\title{
THE OSCILLATING RECTANGULAR AIRFOIL AT SUPERSONIC SPEEDS*
}

\author{
BY \\ JOHN W. MILES** \\ University of California, Los Angeles
}

Summary. The pressure distribution on a quarter infinite, zero thickness airfoil having a prescribed distribution of downwash (on the wing only), which exhibits a harmonic time dependence, is determined by a Fourier transform solution of the linearized, potential equation for supersonic flow. The solution is effected with the aid of the Wiener-Hopf technique and leads to a Green's function, which may be expressed either as a finite, definite integral or as an expansion in powers of a dimensionless frequency parameter. It is shown that the results are applicable to the calculation of the forces and moments on rectangular airfoils of effective aspect ratio $(A \cot \theta$, where $\theta$ is the Mach angle) greater than unity. It appears that the force and moment coefficients of practical interest may be expressed in terms of known functions, including certain integrals which have been calculated for the two-dimensional, oscillating airfoil. The extension of the two-dimensional results to rectangular wings for which the prescribed downwash is constant along the span is particularly simple. The extension of the results for harmonic time dependence to the step function (Heaviside) case is indicated.

1. Introduction. The linearized, two-dimensional problem of the oscillating airfoil at supersonic speeds has been studied by a number of analysts, using a variety of approaches. This work has recently been collected and summarized in two reports prepared by Biot et. al., ${ }^{1,2}$ which give both the methods of analysis ${ }^{1}$ and the numerical results. ${ }^{2}$ (The reports also include the subsonic, compressible case.) These results are probably adequate for the strip theory analysis of wings with supersonic leading and trailing edges and aspect ratios sufficiently high to render tip effects small. (For an approximate treatment of the swept wing, reference may be made to a recent paper by the writer. ${ }^{3}$ ) Unfortunately, those wings which meet the limitation of supersonic leading and trailing edges are generally characterized by small aspect ratio; moreover, the most serious supersonic flutter problems indicated by two dimensional analyses of such wings frequently occur in the near sonic regime, where tip effects are by no means negligible. Accordingly, it is of considerable practical importance to consider the three dimensional problem of the oscillating airfoil.

The problem selected for study in the present paper is that of the rectangular wing tip, since it is the simplest three dimensional configuration (excepting those wings with no subsonic edges) of practical import. The results may be applied directly to the rectangular airfoil of aspect ratio sufficiently large to prevent the Mach waves from the leading edge wing tips from intersecting one another forward of the trailing edge and,

*Received November 21, 1949.

${ }^{* *}$ Consultant, U.S. Naval Ordnance Test Station, Inyokern, Calif. The author wishes to thank Dr. D. E. Zilmer for his careful criticism and helpful suggestions in the preparation of the manuscript.

'J. N. Karp, S. S. Shu, H. Weil, M. A. Biot, Aerodynamics of the oscillating airfoil in compressible flow, F-TR-1167-ND, HQ, AMC, Wright Field, Dayton, Ohio (1947).

'J. N. Karp, H. Weil, M. A. Biot, The oscillating airfoil in a compressible fluid, F-TR-1195-ND (1948).

3J. W. Miles, Harmonic and transient motion of a swept wing in supersonic flow, J. Aero. Sci. 15, 343-347 (1948). 
indirectly, to the case where these Mach waves intersect on the wing but do not intersect the opposite side edges. It is also possible to solve the case of arbitrarily small aspect ratio, but the results are so complex as to be of dubious practical value.

The method of solution to be used follows the Wiener-Hopf technique ${ }^{4}$ which has previously been applied to the rectangular wing in a steady flow. ${ }^{5}$

2. Statement of problem. A thin, rectangular airfoil is located in the vicinity of the plane $z=0$, and its projection there occupies the first quadrant of the $x y$ plane. A flow of supersonic velocity $U$ is directed along the positive $x$ axis, so that the leading edge of the airfoil is projected on the positive $y$ axis and the port side edge on the positive $x$ axis, as shown in Fig. 1. The boundary conditions are linearized in the usual manner, so that they may be applied at the projection of the airfoil on the plane $z=0$, rather

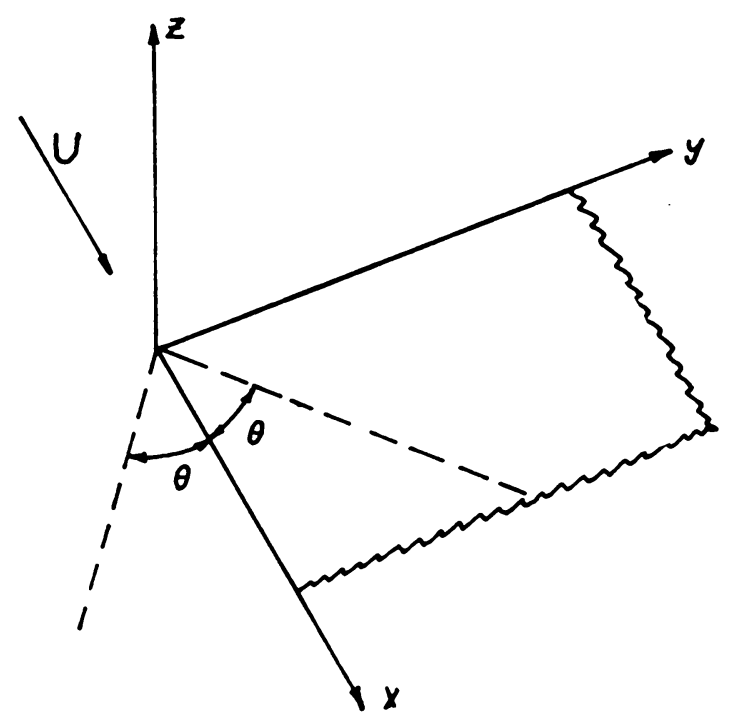

FIG. 1. $x, y, z$ axes and projection of airfoil on $x y$ plane.

than at the airfoil proper. The equations of flow will also be linearized ${ }^{6}$ so that the problem may be subdivided into antisymmetric and symmetric cases with respect to the plane $z=0$; only the former case is of interest here, since the latter situation does not give rise to lift. Accordingly, it is sufficient to consider the half space $z>0$ and to apply the boundary conditions appropriate to the airfoil in the plane $z=0+$. The problem to be solved is then the specification of the perturbation pressure over the first quadrant of the plane $z=0+$ from a knowledge of the downwash there.

${ }^{4}$ N. Wiener and R. Paley, The Fourier transform in the complex domain, Amer. Ma. Soc. Colloq. Publ. 19, Ch. IV (1934); E. C. Titchmarsh, Theory of Fourier integrals, Oxford Press (1937) 339-349; E. Reissner, J. Ma. \& Ph. 20, 219-223 (1941).

5 J. W. Miles, On the rectangular airfoil at supersonic speeds, No. Amer. Avia. Report AL 866 (1948).

${ }^{6} \mathrm{~A}$ more complete discussion of the linearizing process and its various aspects is given by P. A. Lagerstrom, Linearized supersonic theory of conical wings, J.P.L. Progress Report 4-36. California Institute of Technology (1947); NACA T.N. 1685 (1948). 
The vector, perturbation velocity $(\mathbf{q})$ due to the presence of the airfoil in the flow is specified as the gradient of a velocity potential $U b \phi$, viz:

$$
\mathbf{q}(x, y, z, t)=U b \nabla \phi(x, y, z, t)
$$

( $b$ is a characteristic length, to be chosen in any convenient manner.) The gage pressure follows from Newton's law as:

$$
p(x, y, z, t)=-\rho U b \frac{D}{D t} \phi(x, y, z, t)
$$

where $D / D t$ is the time differentiation operator in a fixed reference frame and, in linearized form, is given by:

$$
\frac{D}{D t}=U \frac{\partial}{\partial x}+\frac{\partial}{\partial t}
$$

The condition of continuity, after linearization, leads to the scalar Helmholtz equation in the fixed reference frame, viz.:

$$
\nabla^{2} \phi=\frac{1}{c^{2}} \frac{D^{2}}{D t^{2}} \phi
$$

where $c$ is the sonic velocity for the ambient stream conditions.

At this point, it is convenient to introduce the harmonic time dependence exp $(i \omega t)$, the Mach angle $\theta$, the frequency parameter $\kappa$, the dimensionless coordinates $\left(x^{\prime}, y^{\prime}, z^{\prime}\right)$, and the modified potential, pressure, and downwash functions $\psi\left(x^{\prime}, y^{\prime}, z^{\prime}\right), \gamma\left(x^{\prime}, y^{\prime}\right)$, and $\alpha\left(x^{\prime}, y^{\prime}\right)$, in accordance with the relations

$$
\begin{gathered}
\theta=\sin ^{-1}(c / U) \\
\kappa=(\omega b / c) \tan \theta \\
x=(b \cot \theta) x^{\prime} \\
y=b y^{\prime} \\
z=b z^{\prime} \\
\phi(x, y, z, t)=\exp \left[i\left(\omega t-\kappa x^{\prime} \csc \theta\right)\right] \psi\left(x^{\prime}, y^{\prime}, z^{\prime}\right) \\
\frac{D}{D t} \phi(x, y, 0+, t)=(U \tan \theta / b) \exp \left[i\left(\omega t-\kappa x^{\prime} \csc \theta\right)\right] \gamma\left(x^{\prime}, y^{\prime}\right) \\
\gamma\left(x^{\prime}, y^{\prime}\right)=\left(\frac{\partial}{\partial x^{\prime}}-i \kappa \sin \theta\right) \psi\left(x^{\prime}, y^{\prime}, 0+\right) \\
\alpha\left(x^{\prime}, y^{\prime}\right)=-\frac{\partial}{\partial z^{\prime}} \psi\left(x^{\prime}, y^{\prime}, 0+\right)
\end{gathered}
$$

Substitution of Eq. (2.8) in Eq. (2.4) yields the reduced equation

$$
\psi_{y^{\prime} y^{\prime}}+\psi_{z^{\prime} z^{\prime}}=\psi_{x^{\prime} x^{\prime}}+\kappa^{2} \psi
$$


while substituting Eq. (2.9a) in Eq. (2.2) yields

$$
p(x, y, 0+, t)=-\rho U^{2} \tan \theta \exp \left[i\left(\omega t-\kappa x^{\prime} \csc \theta\right)\right] \gamma\left(x^{\prime}, y^{\prime}\right)
$$

The boundary value problem to be solved now may be posed as: find a solution to Eq. (2.11) which satisfies the boundary conditions

$$
\begin{array}{ll}
\psi_{z^{\prime}}\left(x^{\prime}, y^{\prime}, 0+\right)=-\alpha_{+}\left(x^{\prime}, y^{\prime}\right), & y^{\prime}>0 \\
\gamma\left(x^{\prime}, y^{\prime}\right)=0, & y^{\prime} \leq 0 \\
\psi\left(x^{\prime}, y^{\prime}, z^{\prime}\right) \equiv 0, & x^{\prime}<z^{\prime}
\end{array}
$$

Eq. (2.13) states that the (modified) downwash $\alpha_{+}\left(x^{\prime}, y^{\prime}\right)$, is prescribed on the airfoil; Eq. (2.14) states that the pressure must vanish off the airfoil, since it is presumed asymmetric with respect to $z$, and only the airfoil is capable of supporting a discontinuity in pressure; and Eq. (2.15) states that the disturbance is propagated downstream and must vanish forward of the Mach waves originating at the leading edge of the airfoil.

3. Fourier integral formulation. A general solution to Eq. (2.11) may be conveniently formulated in terms of Fourier integrals. The Fourier transformation of a function of the space coordinates $(x, y)$ into its representative in the $(\mu, \nu)$ spectrum will be denoted by a transition from lower to upper case letters in the functional notation, and the conjugate transform operators $T$ and $\bar{T}$ are defined by

$$
\begin{aligned}
& f(x, y)=T\{F(\mu, \nu)\}=\frac{1}{2 \pi} \int_{-\infty}^{\infty} d \mu \int_{-\infty}^{\infty} d \nu F(\mu, \nu) \exp [i(\mu x+\nu y)] \\
& F(\mu, \nu)=\bar{T}\{f(x, y)\}=\frac{1}{2 \pi} \int_{-\infty}^{\infty} d x \int_{-\infty}^{\infty} d y f(x, y) \exp [-i(\mu x+\nu y)]
\end{aligned}
$$

In general, the parameters $\mu$ and $\nu$ may be allowed complex values, but the paths of integration in the $\mu$ and $\nu$ planes must be suitably restricted in order to comply with both physical and mathematical requirements. Frequent reference will be made to Titchmarsh $^{7}$ and Campbell and Foster ${ }^{8}$, simply by using the letters $T$ and $C F$, followed by the appropriate equation number in the original source, although the notation used herein is not entirely consistent with either of these references. ${ }^{9}$

In addition to the entire transforms of Eq. (3.1), it is expedient to introduce the notation

$$
\begin{aligned}
& F_{+}(\mu, \nu)=\bar{T}\{f(x, y) \mathbf{1}(x, y)\} \\
& F_{-}(\mu, \nu)=\bar{T}\{f(x, y) \mathbf{1}(x,-y)\}
\end{aligned}
$$

${ }^{7}$ E. C. Titchmarsh, Theory of Fourier integrals, Oxford Univ. Press (1937).

${ }^{8}$ G. A. Campbell and R. M. Foster, Fourier integrals for practical applications, Bell Tel. Syst. Mono. B-584 (1942); also published by D. Van Nostrand and Co., New York, N. Y. (1948).

${ }^{9}$ In the case of transformation with respect to a single variable, we find it convenient, however, to use the notation of ref. 8, such that, e.g. $f(x)=T_{\mu}\{F(\mu)\}=1 / 2 \pi \int^{\infty}{ }_{-\infty} d \mu F(\mu) \exp (i \mu x)$.

Thus, the inversions of section 5 correspond to ref. 8 . 


$$
\begin{array}{rrrr}
1(x, y)=1, & x>0 & \text { and } & y>0 \\
0, & x<0 & \text { or } & y<0
\end{array}
$$

Eq. (3.3) defines the Heaviside step function in two variables, but if only one variable is indicated, e. g., $1(x)$, the step is in that variable alone. $F_{+}(\mu, \nu)$ then represents the transform of a function which vanishes off the wing, while $F_{-}(\mu, \nu)$ represents the transform of a function which vanishes on the wing, recalling, cf. Eq. (2.15), that the solution vanishes identically forward of the wing.

It is readily shown that an elementary solution to Eq. (2.11) is given by

$$
\begin{gathered}
\psi_{0}(x, y, z)=\exp [i(\mu x+\nu y)-\lambda z] \\
\lambda=\left[\nu^{2}-\left(\mu^{2}-\kappa^{2}\right)\right]^{1 / 2}
\end{gathered}
$$

the sign of the exponent $\lambda z$ being chosen to represent a disturbance which is bounded for large $z$. The primes have been dropped from the coordinates, but they are assumed to be the dimensionless (primed) coordinates of Eq. (2.7). By virtue of the linearization of the problem, these elementary solutions may be synthesized with the aid of the Fourier integral to form solutions capable of satisfying prescribed boundary conditions. In particular, the most general solution to Eq. (2.11) reducing to $\gamma(x, y)$, cf. Eqs. (2.9b) and (2.14), is given by

$$
\psi(x, y, z)=T\left\{\frac{\Gamma_{+}(\mu, \nu) \exp (-\lambda z)}{i(\mu-\kappa \sin \theta)}\right\}
$$

provided that the paths of integration in the $\mu$ and $\nu$ planes are suitably chosen.

In order to determine the appropriate paths in the complex transform planes, it is necessary to establish a domain of regularity for $\Gamma_{+}(\mu, \nu)$. If it is simply assumed that $\gamma(x, y)$ is bounded for large $x$ (the behavior of $\gamma$ in $y$ affects the behavior of $\Gamma$ in $\nu$ only), it follows from Eq. (3.1b) that $\Gamma_{+}(\mu, \nu)$ will be regular in $\operatorname{Im}(\mu)<0$, so that the $\mu$ integration may be carried out along a path in the lower half of the complex $\mu$ plane. More specifically, $\Gamma_{+}(\mu, \nu)$ will be found to have a simple pole at $\mu=+(\kappa \csc \theta+i \epsilon)$ if the pressure on the wing, cf. Eq. (2.9a) behaves as exp $(-\epsilon x)$ for large $x$, where $\epsilon$ is a positive real constant, and will have a zero at $\mu=\kappa \sin \theta$, as may be verified a posteriori. (Due to the zero in $\Gamma$ at $\mu=\kappa \sin \theta, \Psi$ does not have a pole there.) In the present analysis, it suffices to take $\epsilon=0$, insofar as $\operatorname{Im}(\mu)<0$. Accordingly, the integrand in Eq. (3.6) is regular in $\mu$ except for a simple pole on the real axis and the branch points in $\lambda$, the latter being designated as $\pm \mu_{0}(\nu)$, where, cf. Eq. (3.5),

$$
\begin{gathered}
\lambda=\left(\mu_{0}^{2}-\mu^{2}\right)^{1 / 2} \\
\mu_{0}(\nu)=\left(\kappa^{2}+\nu^{2}\right)^{1 / 2}
\end{gathered}
$$

The location of these branch points of course depends on $\nu$, but if the path in the $\nu$ plane is chosen such that

$$
|\operatorname{Im}(\nu)|<\kappa
$$

they will always possess real parts, and their imaginary parts will always be less (in magnitude) than $|\operatorname{Im}(\nu)|$. It follows that, if the branch cuts from $\pm \mu_{0}$ are both extended 
to $+i \infty$, the integrand of Eq. (3.6) will be regular everywhere in the half plane bounded by

$$
\operatorname{Im}(\mu)<-|\operatorname{Im}(\nu)|<-\left|\operatorname{Im}\left(\mu_{0}\right)\right|
$$

and the phase of $\lambda$ will be $\pi / 2,0$, and $-\pi / 2$ for $\operatorname{Im}(\mu)=0$ and $\operatorname{Re}(\mu)>\mu_{0}$, $|\operatorname{Re}(\mu)|<\mu_{0}$ and $\operatorname{Re}(\mu)<-\mu_{0}$, respectively. An appropriate path of integration is then one satisfying the restriction (3.10), as illustrated in Fig. 2.

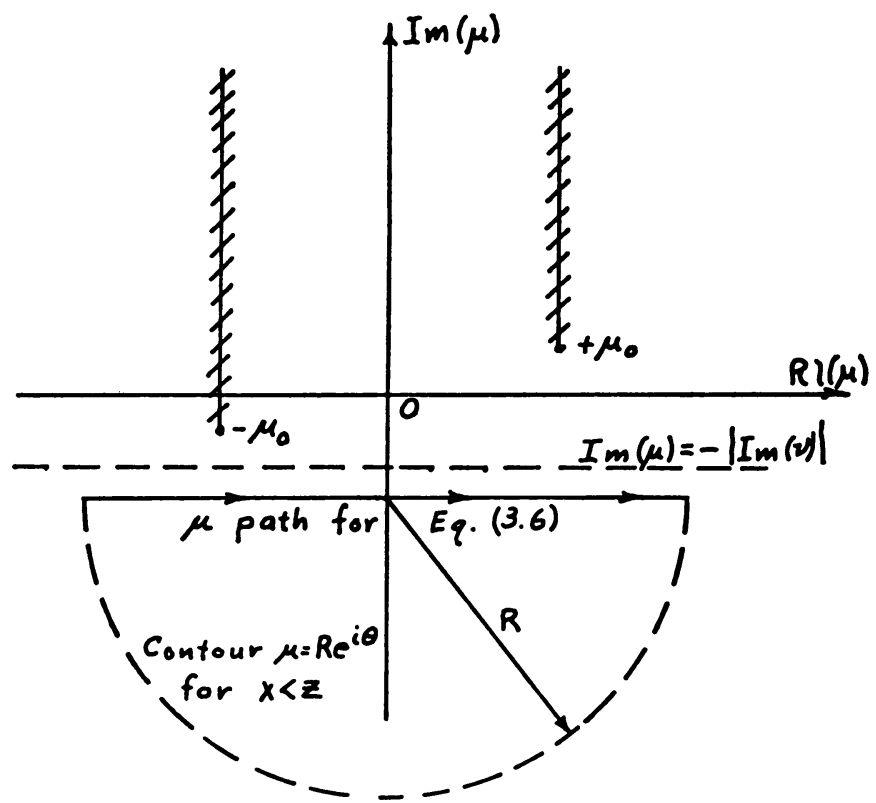

FIG. 2. $\mu$ plane, showing branch points $\pm \mu_{0}$ in $\lambda$, the branch cuts therefrom, the path of integration for Eq. (3.6), and the completion of this path for $x<z$.

Consider now the behavior of the solution (3.6) for $x<z$. The path of integration along the horizontal line chosen in accordance with Eq. (3.10) may be closed by a semicircle in the lower half $(\mu)$ plane. If $\mu$ is denoted by $\operatorname{Re}^{i \theta}$ along the latter path, the choice of phase for $\lambda$ implies

$$
|\exp (i \mu x-\lambda z)| \sim|\exp [i \mu(x-z)]| \sim \exp [-R(x-z) \sin \theta]
$$

Since $\theta$ is negative along the semi-circle, the integrand of Eq. (3.6) is exponentially bounded, as long as $x<z$, and the contribution of this path to the contour integral therefore vanishes uniformly as $R$ is allowed to become infinite (for $-\pi+\epsilon<\theta<-\epsilon$, $\epsilon>0$; the portions $-\epsilon<\theta<0$ and $-\pi<\theta<-\pi+\epsilon$ may be interpreted as belonging to the path associated with the transform path for $\psi$ ). Moreover, since the integrand is regular in the half plane bounded by Eq. (3.10), the entire contour integral vanishes by virtue of Cauchy's theorem, whence $\psi(x, y, z)$ vanishes identically for $x<z$, in satisfaction of Eq. (2.15).

The asymptotic behavior of the solution for large, positive $x$ (i.e., the Trefftz plane) may be obtained by closing the contour in $\operatorname{Im}(\mu)>0$ plus "keyhole" paths around the 
branch points $\pm \mu_{0}$, the only contributions to the integral coming from the latter paths and the pole at $\mu=+\kappa \csc \theta .^{10}$

Turning to the $\nu$ plane, the branch points of $\lambda$ may be designated as $\pm \nu_{0}$, where

$$
\begin{gathered}
\lambda=\left(\nu^{2}-\nu_{0}^{2}\right)^{1 / 2} \\
\nu_{0}(\mu)=\left(\mu^{2}-\kappa^{2}\right)^{1 / 2}
\end{gathered}
$$

Considering $\nu_{0}(\mu)$ in the $\mu$ plane, the branch cuts are taken from $\pm \kappa$ to $\pm \infty$, so that the phase of $\nu_{0}$ will be $0,-\pi / 2$, and $-\pi$ for $\operatorname{Im}(\mu)=0$ and $\operatorname{Re}(\mu)>\kappa,|\operatorname{Re}(\mu)|<\kappa$,

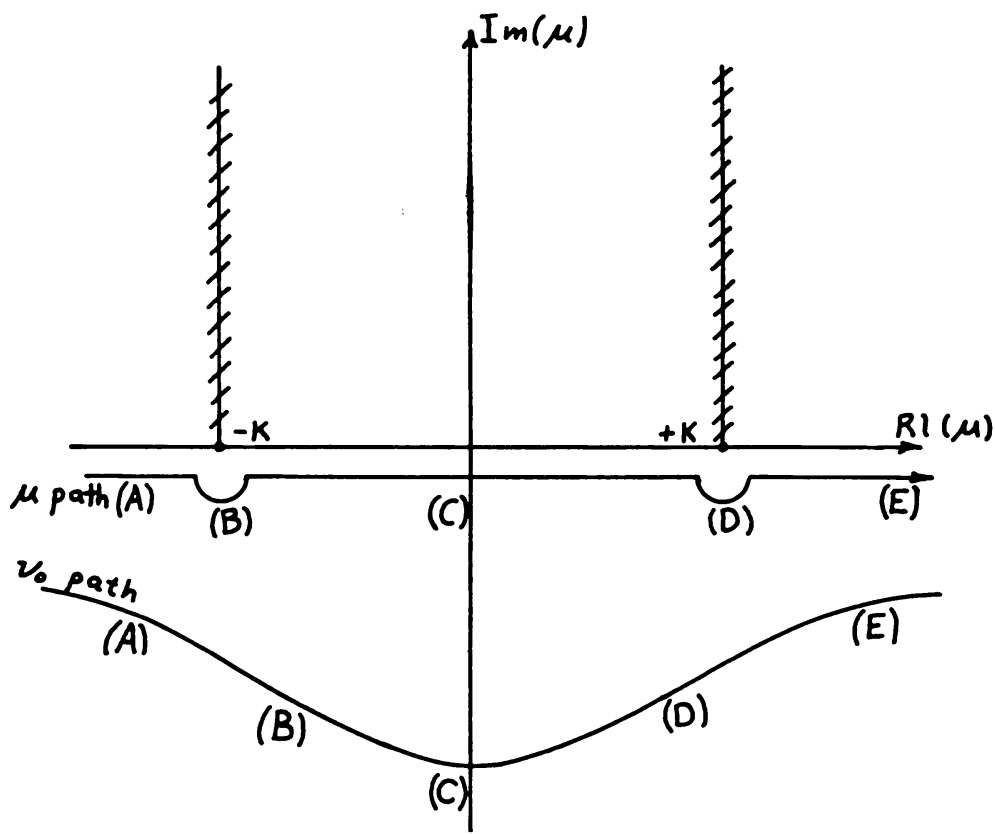

Fig. 3. $\mu$ plane, showing cuts from $\pm \kappa$ and paths of $\mu$ and $\nu_{0}(\mu) . A, B, C, D, E$, represent corresponding points on these paths.

and $\operatorname{Re}(\mu)<-\kappa$, respectively. As $\mu$ follows the horizontal path designated by Eq. (3.10) $\nu_{0}(\mu)$ will follow a lower path, as shown in Fig. 3, such that

$$
\operatorname{Im}\left(\nu_{0}\right)<\operatorname{Im}(\mu)
$$

The cuts from the branch points $\pm \nu_{0}$ in the $\nu$ plane must be chosen such that $\operatorname{Re}(\lambda) \geq 0$ (in order that the solution (3.6) will be bounded for all positive $z$ ) for all $(\mu, \nu$ ); accordingly, the path of integration in the $\nu$ plane must pass under one branch point and over

${ }^{10}$ If the path for Eq. (3.6) is allowed to approach the real axis (also taking $\operatorname{Im}(\nu)=0$ ) and is indented under $+\kappa \csc \theta$ and $干_{\mu_{0}}$, the pole will give only half the contribution obtained for $\operatorname{Im}(\mu)<0$ and will also contribute an equal and opposite amount to the contour for $x<z$. It is necessary in this case to add an auxiliary solution, which is independent of $x$ and is represented by $\pi \delta(\mu-\kappa \csc \theta) \Gamma_{+}(\kappa \csc \theta, \nu)$ - $\exp \left[-\left(\nu^{2}-\kappa^{2} \cot ^{2} \theta\right)^{1 / 2} z\right]$. See, e.g., J. W. Miles, On linearized supersonic airfoil theory, No. Amer. Avia., Inc. Report AL-801 (1948) pp. 15, 16, where the care of stationary flow $(\kappa=0)$ is discussed. 
the other, and, in addition, the cuts must not interfer as Re $\left(\nu_{0}\right)$ changes sign, cf. Figs. 3 . and 4. It follows that the cuts must run from $+\nu_{0}$ to $-i \infty$ and from $-\nu_{0}$ to $+i \infty$, since $\operatorname{Im}\left(\nu_{0}\right) \leq 0$ for all $\mu$, as shown in Fig. 4. The result of this choice is that the elementary solution $\psi_{0}(x, y, z)$, cf. Eq. (3.4), behaves as $\exp [i \mu(x-z)]$ for large (absolute) values of $\mu$ and not too large values of $\nu$, indicating the (Mach) wave front $x=z$, representing the locus of the waves originating at the leading edge of the wing. ${ }^{11}$

Returning to the solution (3.6), differentiating with respect to $z$, and substituting in Eq. (2.13) yields

$$
\begin{aligned}
& \alpha_{+}(x, y)=T\left\{G(\mu, \nu) \Gamma_{+}(\mu, \nu)\right\}, \quad y>0 \\
& G(\mu, \nu)=-i(\mu-\kappa \sin \theta)^{-1}\left[\nu^{2}-\nu_{0}^{2}(\mu)\right]^{1 / 2}
\end{aligned}
$$

It may be remarked that Eq. (3.15) is valid for all $y$ if $\alpha_{-}(x, y)$ is added to $\alpha_{+}(x, y)$.

Eq. (3.15), for $y>0$, and Eq. (2.14), for $y<0$, together constitute a dual integral

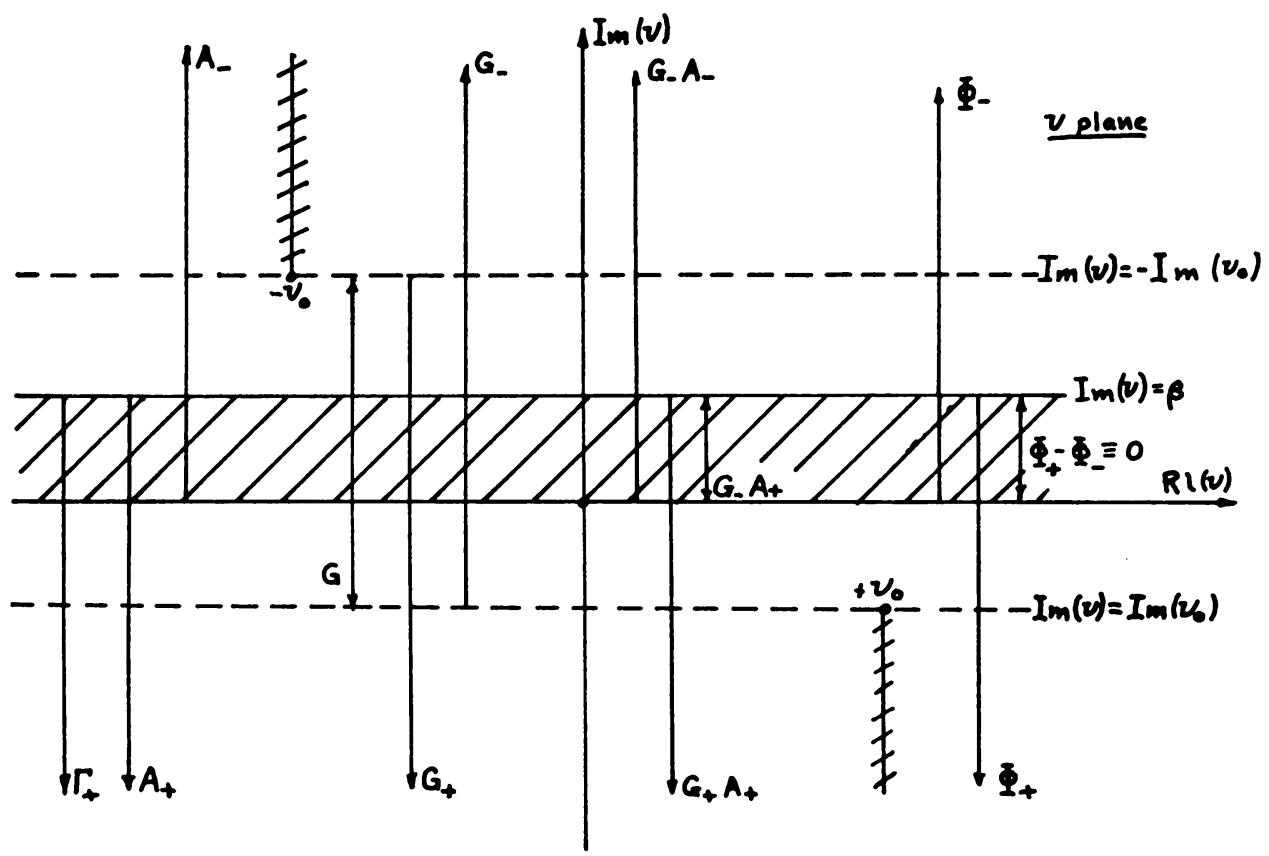

Fig. 4. $\nu$ plane, showing cuts from $\pm \nu_{0}$ and the domains of regularity of the various transforms.

equation (cf. $T$, pp. 334-342) for the determination of $\gamma(x, y)$. Its reduction to transform equations may be effected by taking the inverse transforms of Eqs. (3.15), extended as valid for all $y$, and (2.14). The results are

$$
\begin{gathered}
G(\mu, \nu) \Gamma_{+}(\mu, \nu)=A_{+}(\mu, \nu)+A_{-}(\mu, \nu) \\
\Gamma_{-}(\mu, \nu)=0
\end{gathered}
$$

${ }^{11}$ See also Eq. (3.11) in this respect. 
4. Solution of transform equation. The transform equation (3.17) may be solved with the aid of the Wiener-Hopf technique. While it is possible to treat an arbitrary distribution $\alpha_{+}(x, y)$, the difficulty of inverting the complicated transforms which arise in any general solution probably would render it impracticable. Accordingly, it will be assumed that

$$
\alpha_{+}(x, y)=y^{n} \exp (-\beta y) \mathbf{1}(y) \alpha_{n}(x)
$$

which will be sufficiently general for most purposes.

The transform of Eq. (4.1) with respect to $y$ is given by CF 524.2, while the transform with respect to $x$ will be denoted by $A_{n}(\mu),{ }^{12}$ whence

$$
\begin{aligned}
& A_{+}(\mu, \nu)=(2 \pi)^{-1} \Gamma(n+1)(\beta+i \nu)^{-n-1} A_{n}(\mu) \\
& A_{n}(\mu)=T_{\mu}\left\{\alpha_{n}(\xi)\right\}=\int_{0}^{\infty} d \xi \exp (-i \mu \xi) \alpha_{n}(\xi)
\end{aligned}
$$

Accordingly, $A_{+}(\mu, \nu)$ is regular in $\nu$ except for a pole of order $n+1$ at $\nu=i \beta$. Moreover, it may be verified a posteriori that $\gamma(x, y)$ behaves as $\alpha_{+}(x, y)$ for large $y$ and, therefore, also has a pole of order $n+1$ at $\nu=i \beta$, while the fact that $\gamma(x, y)$ vanishes for $y=0$, cf. Eq. (2.14), implies that its transform must vanish at least as $\nu^{-1-\epsilon}$, where $\epsilon$ is a positive constant, for large $\nu$. Similarly, it may be verified that $\alpha_{-}(x, y)$ has a singularity of at worst $y^{-1+}$ for small (negative) $y$ and vanishes uniformly for large $y$, so that its transform vanishes for both large and small $y$. It follows that sufficient conditions for regularity of the various transforms in the complex $\mu$ and $\nu$ planes, and, therefore, suitably restricted domains in these planes, are

$$
\begin{array}{lll}
A_{+}(\mu, \nu): & \operatorname{Im}(\mu)<-\beta, & \operatorname{Im}(\nu)<\beta \\
A_{-}(\mu, \nu): & \operatorname{Im}(\mu)<-\beta, & \operatorname{Im}(\nu)>0 \\
\Gamma_{+}(\mu, \nu): & \operatorname{Im}(\mu)<-\beta, & \operatorname{Im}(\nu)<\beta \\
G(\mu, \nu): & \operatorname{Im}(\mu)<-\beta, & |\operatorname{Im}(\nu)|<\left|\operatorname{Im}\left(\nu_{0}\right)\right|>\beta
\end{array}
$$

The restriction $\operatorname{Im}(\mu)<-\beta$ is in accordance with Eq. (3.10) and conveniently places the line $\operatorname{Im}(\nu)=-\operatorname{Im}\left(\nu_{0}\right)$ above $\operatorname{Im}(\nu)=\beta$, as shown in Eq. 4 . It should be remarked that these conditions refer only to the individual transforms and do not necessarily imply regularity of the complete transform in Eq. (3.6), for which it is sufficient to require Eqs. (3.9) and (3.10).

Since $A_{+}$and $\Gamma_{+}$are regular in the lower half $\nu$ plane and $A_{-}$in the upper half, it is expedient to split $G$ into two functions, which are regular, respectively, in these two domains; thus:

$$
\begin{gathered}
G(\mu, \nu)=G_{+}(\mu, \nu) / G_{-}(\mu, \nu) \\
G_{+}(\mu, \nu)=-i(\mu-\kappa \sin \theta)^{-1}\left(\nu+\nu_{0}\right)^{1 / 2}, \quad \operatorname{Im}(\nu)<-\operatorname{Im}\left(\nu_{0}\right) \\
G_{-}(\mu, \nu)=\left(\nu-\nu_{0}\right)^{-1 / 2}, \quad \operatorname{Im}(\nu)>\operatorname{Im}\left(\nu_{0}\right)
\end{gathered}
$$

${ }^{12}$ See footnote 9 regarding notation. 
It should be specifically noted that $G_{+}$and $G_{-}$are not defined in accordance with Eq. (3.2).

The domains of regularity of $A_{+}, A_{-}, \Gamma_{+}, G, G_{+}$, and $G_{-}$in the $\nu$ plane are shown in Fig. 4. Moreover, if Eq. (3.17a) is multiplied through by $G_{-}(\mu, \nu)$, it is evident that

$$
\begin{array}{lll}
G_{-}(\mu, \nu) A_{-}(\mu, \nu) & \text { is regular in } & \operatorname{Im}(\nu)>0 \\
G_{+}(\mu, \nu) A_{+}(\mu, \nu) & \text { is regular in } & \operatorname{Im}(\nu)<\beta \\
G_{-}(\mu, \nu) A_{+}(\mu, \nu) & \text { is regular in } & 0<\operatorname{Im}(\nu)<\beta
\end{array}
$$

Since $A_{+}(\mu, \nu)$ is regular throughout the entire $\nu$ plane except for the pole of order $(n+1)$ at $\nu=i \beta$, it is possible to remove the singular part of $G_{-}(\mu, \nu) A_{+}(\mu, \nu)$ to obtain a function which is regular in the upper half plane. Thus, introducing a Taylor expansion for $G_{-}(\mu, \nu)$ about $\nu=i \beta$,

$$
\begin{array}{r}
G_{-}(\mu, \nu) A_{+}(\mu, \nu)=\left[\sum_{m=0}^{n} \Gamma^{-1}(m+1) G_{-}^{(m)}(\mu, i \beta)(\nu-i \beta)^{m}\right] A_{+}(\mu, \nu) \\
+\left[G_{-}(\mu, \nu)-\sum_{m=0}^{n} \Gamma^{-1}(m+1) G_{-}^{(m)}(\mu, i \beta)(\nu-i \beta)^{m}\right] A_{+}(\mu, \nu)
\end{array}
$$

it is found that the second term on the right is $0(1)$ at $\nu=i \beta$, while the first term remains $0\left[(\nu-i \beta)^{-n-1}\right]$, and it follows that the two terms are regular in the upper and lower half $\nu$ planes, respectively. Multiplying Eq. (3.17a) through by $G_{-}(\mu, \nu)$ and subtracting from both sides the second term on the right of Eq. (4.4), the result may be written

$$
\begin{gathered}
\Phi_{+}(\nu) \equiv \Phi_{-}(\nu), \quad 0<\operatorname{Im}(\nu)<\beta \\
\Phi_{+}(\nu)=G_{+}(\mu, \nu) \Gamma_{+}(\mu, \nu)-\left[\sum_{m=0}^{n} \Gamma^{-1}(m+1) G_{-}^{(m)}(\mu, i \beta)(\nu-i \beta)^{m}\right] A_{+}(\mu, \nu), \\
\Phi_{-}(\nu)=G_{-}(\mu, \nu) A_{-}(\mu, \nu)+\left[G_{-}(\mu, \nu)\right. \\
\left.-\sum_{m=0}^{n} \Gamma^{-1}(m+1) G_{-}^{(m)}(\mu, i \beta)(\nu-i \beta)^{m}\right] A_{+}(\mu, \nu), \quad \operatorname{Im}(\nu)>0
\end{gathered}
$$

where $\Phi_{+}(\nu)$ and $\Phi_{-}(\nu)$ are regular in the indicated domains, are identical in the common strip of regularity $0<\operatorname{Im}(\nu)<\beta$, and are, therefore, analytic continuations of the same function, say $\Phi(\nu)$, in the lower and upper half planes, respectively.

The problem is now reduced to the determination of the function $\Phi(\nu)$, and, since $\Phi(\nu)$ is regular for all $\nu$, it must be an integral function. ${ }^{13}$ Indeed, since it is regular at infinity as well as in the finite plane, it must be a constant, by virtue of Liouville's theorem. ${ }^{14}$ To determine this constant, it suffices to examine $\Phi(\nu)$ for large $\nu$. Recalling

${ }^{13}$ E. T. Whittaker and G. N. Watson, Modern analysis, Macmillan Co., New York (1947), p. 105.

${ }^{14}$ Ibid. 
that $\Gamma_{+}(\mu, \nu)$ must vanish at least as $\nu^{-1}$, that $A_{+}(\mu, \nu)$ is of order $\nu^{-n-1}$, and that $G_{+}(\mu, \nu)$ and $G_{-}(\mu, \nu)$ are $0\left(\nu^{1 / 2}\right)$ and $0\left(\nu^{-1 / 2}\right)$, respectively, it follows that $\Phi(\nu)$ is $0\left(\nu^{-1 / 2}\right)$ and therefore vanishes identically.

Substituting the result $\Phi=0, G_{+}$and $G_{-}$from Eq. (4.3), $A_{+}$from Eq. (4.2), and evaluating the derivative of $G_{-}(\mu, \nu)$, viz.

$$
G_{-}^{(m)}(\mu, \nu)=(-)^{m} \pi^{-1 / 2} \Gamma(m+1 / 2)\left(\nu-\nu_{0}\right)^{-m-1 / 2}
$$

the results for $\Gamma_{+}$and $A_{-}$are given by Eqs. (4.6) and (4.7) as

$$
\begin{aligned}
& \Gamma_{+}(\mu, \nu)=i(2 \pi)^{-1} \pi^{-1 / 2}(\mu-\kappa \sin \theta)\left(\nu+\nu_{0}\right)^{-1 / 2}\left(i \beta-\nu_{0}\right)^{-1 / 2} \Gamma(n+1) A_{n}(\mu) \\
& \cdot \sum_{m=0}^{n} \Gamma^{-1}(m+1) \Gamma(m+1 / 2)\left(\frac{\nu-i \beta}{\nu_{0}-i \beta}\right)^{m}(\beta+i \nu)^{-n-1} \\
& A_{-}(\mu, \nu)=(2 \pi)^{-1} \pi^{-1 / 2}\left(\nu-\nu_{0}\right)^{1 / 2}\left(i \beta-\nu_{0}\right)^{-1 / 2} \Gamma(n+1) A_{n}(\mu) \\
& \cdot \sum_{m=0}^{n} \Gamma^{-1}(m+1) \Gamma(m+1 / 2)\left(\frac{\nu-i \beta}{\nu_{0}-i \beta}\right)^{m}(\beta+i \nu)^{-n-1}-A_{+}(\mu, \nu)
\end{aligned}
$$

This completes the solution of the transform equation (3.17), and the remainder of the paper will be devoted primarily to the pressure distribution, as represented by $\Gamma_{+}(\mu, \nu)$. A similar treatment may be accorded $A_{-}(\mu, \nu)$ to obtain the downwash off the wing, but it is of less practical importance.

In interpreting the result for $\Gamma_{+}(\mu, \nu)$, it is convenient to regroup the terms and write, cf. Eq. (2.9b),

$$
\begin{gathered}
\Gamma_{+}(\mu, \nu)=i(\mu-\kappa \sin \theta) \Psi(\mu, \nu) \\
\Psi(\mu, \nu)=(2 \pi)^{-1} \pi^{-1 / 2}\left(i \nu+i \nu_{0}\right)^{-1 / 2} \Gamma(n+1) A_{n}(\mu) \\
\cdot \sum_{m=0}^{n} \Gamma^{-1}(m+1) \Gamma(m+1 / 2)\left(\beta+i \nu_{0}\right)^{-m-1 / 2}(\beta+i \nu)^{m-n-1}
\end{gathered}
$$

where $\Psi(x, y)$ represents the potential only in the sense of Eq. $(2.9 \mathrm{~b}) .{ }^{15}$

In principle, the problem is now reduced to quadrature, i.e. the inversion of the transform $\Psi(\mu, \nu)$. Before considering this inversion, it is of interest to infer the behaviour of $\gamma(x, y)$ for small and large $y$ directly from the behavior of $\Gamma_{+}(\mu, \nu)$ for large and small $\nu$, respectively. Thus, it is found that the pressure behaves as $y^{1 / 2}$ for small $y$, since its transform behaves as $\nu^{-3 / 2}$ for large $\nu$, while it behaves as $y^{n} \exp (-\beta y)$ for large $y$, since the transform behaves as $(\beta+i \nu)^{-n-1}$ for small $\nu$, and the initial assumptions are verified. Similarly, it is found that $\alpha_{-}(x, y)$ behaves as $y^{-1 / 2}$ for small $y$ and as $((x+y) /-y)^{1 / 2} 1(x, y)$ for large $y$, therefore vanishing outside the Mach line $x=-y$ and behaving as $(-y)^{-1 / 2}$ for large (negative) values of $y$ within this Mach line, again verifying the initial assumptions.

\footnotetext{
${ }^{15}$ Inasmuch as the pressure distribution over the wing is independent of the discontinuity of $\psi$ across the vortex sheet aft of the trailing edge (i.e., the "wake"), it would have been possible to formulate the entire boundary value problem in terms of $\psi$. This would not be possible for a wing with a subsonic trailing edge.
} 
5. Pressure distribution. It appears that Eq. (4.12) cannot be inverted in finite terms of known functions. However, the result can be partially inverted so as to exhibit characteristic features, after which it will suffice to deal with certain integrals of the pressure.

The $\nu$ inversion of Eq. (4.12) may be effected by CF 524.2, CF 526, and the Faltung theorem. The end result is

$$
\begin{gathered}
\psi(x, y)=\pi^{-1} 1(y) \sum_{m=0}^{n}\left(\begin{array}{c}
n \\
m
\end{array}\right) \Gamma(m+1 / 2) \int_{0}^{x} d \xi \alpha_{n}(x-\xi) \\
\cdot \int_{0}^{y} d \eta(y-\eta)^{n-m} \eta^{-1 / 2} k_{m}(\xi, \eta) \\
k_{m}(\xi, \eta)=T_{\mu}\left\{\left(i \nu_{0}\right)^{-m-1 / 2} \exp \left(-i \nu_{0} y\right)\right\}
\end{gathered}
$$

where $\left(\begin{array}{l}n \\ m\end{array}\right)$ is the binomial coefficient, and Eq. (5.2) utilizes the transform convention of footnote 9. Eq. (5.2) may be inverted with the aid of CF 571, CF 866, and the Faltung theorem, yielding

$$
\begin{gathered}
k_{m}(x, y)=\pi^{+1 / 2} \Gamma^{-1}\left(\frac{1}{2} m-\frac{1}{4}\right) 1(x, x-y) \int_{\nu}^{x} d \xi[(x-\xi) / 2 \kappa]^{m / 2-3 / 4} \\
\cdot J_{(m / 2-3 / 4)}[\kappa(x-\xi)] J_{0}\left[\kappa\left(\xi^{2}-y^{2}\right)^{1 / 2}\right]
\end{gathered}
$$

While Eq. (5.3) appears too complex for direct applications, it establishes the useful fact that $k_{m}(x, y)$ vanishes for $x<0$ or $y>x$.

By virtue of the fact that $k_{m}(\xi, \eta)$ vanishes for $\eta>\xi$, the upper limit $y$ in Eq. (5.1) may be replaced by infinity in the region where $y>x$. Then, if $(y-\eta)^{n-m}$ is expanded by the binomial theorem, and the $\eta$ integration carried out prior to the $\mu$ inversion, the latter may be effected by CF 571 and the end result expressed in terms of powers of $y$ and integrals of Bessel functions of integral and half integral order. This solution is, however, readily obtainable by more direct methods, since the region in question is not influenced by the region between the side edge and the Mach line $y=-x$.

For small values of $y$ the $\nu$ inversion of Eq. (4.12) may be brought about by expanding the transform in powers of $(1 / i \nu)$, but the results would still involve Bessel functions of fractional (odd multiples of $1 / 4$ ), therefore being only slightly less complicated than the result of Eq. (5.3).

Still another approach is to expand the solution in powers of the frequency parameter $\kappa$. Thus, if we determine the coefficients $c_{p q}^{(m)}$ in the expansion

$$
\begin{aligned}
& \left(i \nu_{0}\right)^{-m-1 / 2} \exp \left(-i \nu_{0} y\right) \\
& \quad=(i \mu)^{-m-1 / 2} \exp (-i \mu y) \sum_{p=0}^{\infty} \kappa^{2 p} \sum_{q=0}^{p} c_{p q}^{(m)}(i \mu)^{a-2 p} y^{a}
\end{aligned}
$$

the $\mu$ inversion of Eq. (5.2) is given by CF 516 as

$$
\begin{gathered}
k_{m}(x, y)=1(y, x-y) \sum_{p=0}^{\infty} \kappa^{2 p} \sum_{q=0}^{p} c_{p a}^{(m)} \Gamma^{-1}\left(2 p-q+m+\frac{1}{2}\right) \\
\cdot y^{a}(x-y)^{2 p-a+m-1 / 2}
\end{gathered}
$$


If only the first term $(p=0)$ in this expansion is retained, the summation in Eq. (5.1) is binomial, and the result may be written

$$
\begin{gathered}
\psi(x, y)=\pi^{-1} 1(y, x-y) \int_{0}^{x} d \xi \int_{0}^{y} d \eta \eta^{-1 / 2}(\xi-\eta)^{-1 / 2} \\
\cdot \alpha(x-\xi, y+\xi-2 \eta) 1(\xi-\eta)+0\left(\kappa^{2}\right)
\end{gathered}
$$

in agreement with the result obtained in footnote reference 5 .

6. Wings of finite aspect ratio. Consider the rectangular planform depicted in Fig. 5,

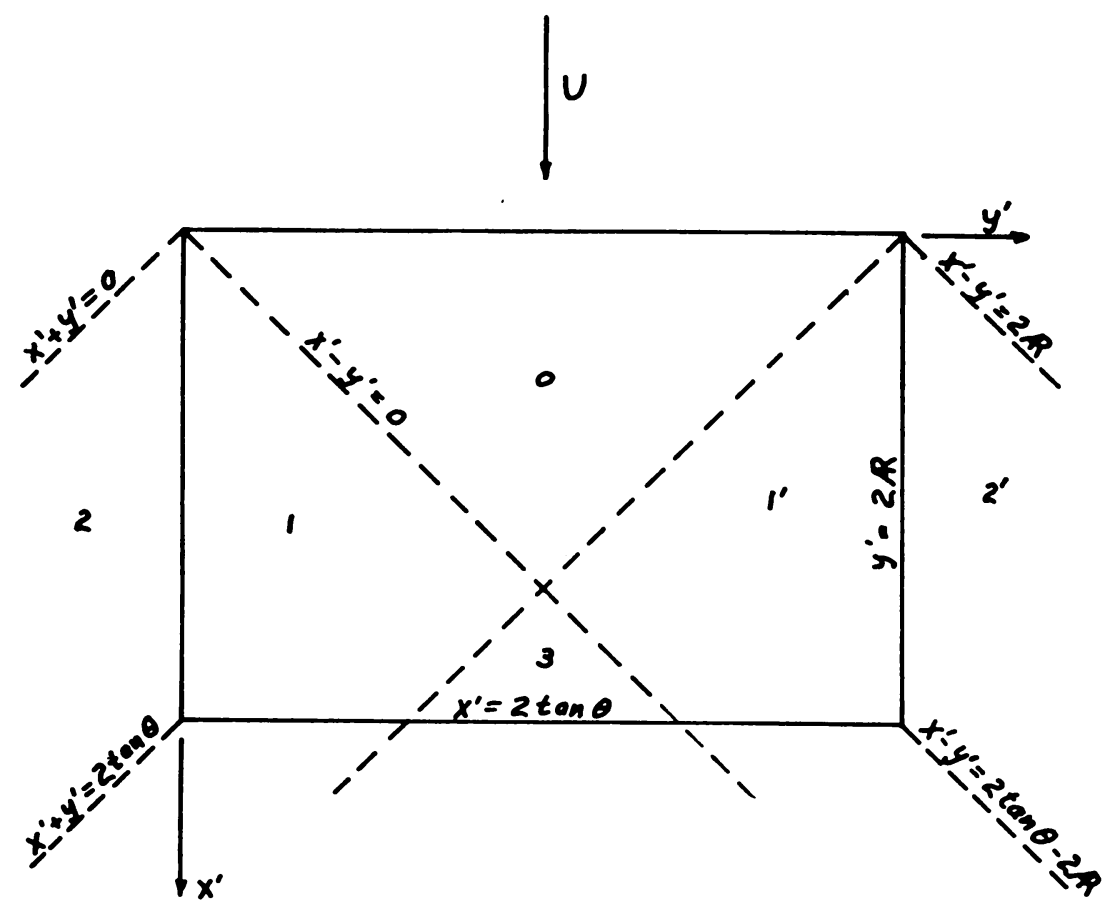

Fig. 5. Rectangular planform and Mach waves.

along with the Mach waves springing from the corners and the regions delineated by these waves. In the dimensionless coordinates of Eq. (2.7), the chord of the wing is chosen as 2 and the span as $2 A$. $A^{\prime}$, the "effective" aspect ratio, is related to the true aspect ration $(A)$ through

$$
A^{\prime}=A \cot \theta
$$

Due to the choice of the dimensionless coordinates, the Mach waves (or lines) make angles of $45^{\circ}$ with the direction of the free stream flow.

A point in region 0 is influenced only by points on the wing, since the fore Mach lines through such a point both intersect the leading edge, so that the downwash is known at every point in the zone of influence of such a point, and the pressure may then be determined by an integration of this downwash. The zone of influence of a point in region I, however, includes part of the region II, off the wing, where the downwash is 
not directly prescribed, at least in the problem under consideration. The effect of this latter region is to supply a pressure deficiency in region $\mathrm{I}$, which is just sufficient to cancel the excess pressure along the side edge $y=0$. Accordingly, if $c_{p_{0}}(x, y)$ denotes the pressure (jump) coefficient at $(x, y)$ due to the downwash at points on the wing which lie in the fore Mach cone subtended from $(x, y)$, and $c_{p_{i}}(x, y)$ represents the pressure deficiency due to the points in region II within this Mach cone, the pressure in region 0 is determined by $c_{p_{0}}(x, y)$ alone, $c_{p_{i}}(x, y)$ vanishing there, while the pressure in region I is determined by

$$
c_{p_{I}}(x, y)=c_{p_{0}}(x, y)-c_{p_{i}}(x, y)
$$

Now, in the case of the quarter infinite wing, the only regions of interest are 0 and I, and the required pressure coefficient indicated by Eq. (6.2) is given by Eq. (5.1), in accordance with the relation, cf. Eq. (2.12),

$$
\begin{aligned}
c_{p}(x, y) & =\left(\rho U^{2} / 2\right)^{-1}[p(x, y, 0-)-p(x, y, 0+)] \\
& =4 \tan \theta \gamma(x, y) \exp [i(\omega t-\kappa x \csc \theta)]
\end{aligned}
$$

That Eq. (6.3) does indeed exhibit a discontinuity (in its derivatives) across the Mach line $x=y$ is indicated by the step function $1(x-y)$, which occurs in the Green's function $k(x, y)$, cf. Eq. (5.3).

The pressure in region $I^{\prime}$ on a rectangular wing of finite span may be determined from the pressure in region I by invoking symmetry considerations. Thus, by virtue of the linearization of the problem, the downwash distribution over the wing may be broken down into symmetric and antisymmetric distributions in accordance with the relations

$$
\begin{aligned}
& \alpha_{+}^{(s)}(x, y)=\alpha_{+}^{(s)}(x, 2 A-y) \\
& \alpha_{+}^{(a)}(x, y)=-\alpha_{+}^{(a)}(x, 2 A-y)
\end{aligned}
$$

and the corresponding pressures determined by $\alpha^{(s)}(x, y)$ and $\alpha^{(a)}(x, y)$ will satisfy similar relations, whence it follows that

$$
c_{p_{1}}(x, y)=c_{p_{0}}(x, y) \mp c_{p_{i}}(x, 2 A-y)
$$

the top and bottom signs being associated with the symmetric and antisymmetric problems, respectively. Accordingly, the pressure at any point on a rectangular wing, for which the Mach lines from the leading edge corners do not intersect on the wing (i.e., $A^{\prime} \geq 2$ ), or indeed for any wing which has the leading edge $x=0$, the side edges $y=0,2 A$, and a trailing edge which lies entirely in the regions $0, \mathrm{I}$, and $\mathrm{I}^{\prime}$ and nowhere has a sweepback angle in excess of the Mach angle ( $45^{\circ}$ in the dimensionless coordinates), is directly determined by the solution for the quarter infinite wing.

Consider, now, the less restricted case for which the Mach lines from the leading edge corners do not intersect the opposite side edges (i.e., $A^{\prime} \geq 1$ ). For this wing, the pressure coefficient in region III may be cast in the form

$$
c_{p_{I I I}}(x, y)=c_{p_{0}}(x, y)-c_{p_{i}}(x, y) \mp c_{p_{i}}(x, 2 A-y)
$$

This result follows directly from superposing the pressure deficiencies from the two edges. If the pressure coefficient given by Eq. (6.6) is now integrated over the wing 
with a weighting factor which is restricted in its $(x, y)$ dependence to have the same symmetry as $\alpha_{+}(x, y)$, it is found that the result is identical with that which would have been obtained on the assumption $A^{\prime} \geq 2$. It follows that the results of this paper may be used for the calculation of the forces and moments on a rectangular wing subject to the restriction $A^{\prime} \geq 1$.

7. Lift and moments. The integrals of primary interest for the oscillating wing are the lift, mid-chord pitching moment, and rolling moment. The appropriate, dimensionless coefficients are defined as follows:

$$
\begin{gathered}
C_{L}=\left[\frac{1}{2} \rho U^{2} S \exp (i \omega t)\right]^{-1} \iint_{S}[-2 p(x, y, 0+)] d x d y \\
C_{M}=\left[\frac{1}{2} \rho U^{2} S \cdot 2 b \cdot \exp (i \omega t)\right]^{-1} \iint_{S}(b-x)[-2 p(x, y, 0+)] d x d y \\
C_{l}=\left[\frac{1}{2} \rho U^{2} S \cdot 2 b A \cdot \exp (i \omega t)\right]^{-1} \iint_{S}(b A-y)[-2 p(x, y, 0+)] d x d y
\end{gathered}
$$

where $(x, y)$ are the true coordinates, $S$ is the wing area, $2 b$ is the chord, and $2 b A$ is the span.

Whereas it was more convenient in studying solutions to the potential equation to deal with the quantities defined in Eqs. (2.5)-(2.10), it is rather more natural in dealing with the forces on the rectangular wing to introduce the new, dimensionless quantities

$$
\begin{gathered}
x^{\square}=x / 2 b=1 / 2 x^{\prime} \cot \theta \\
y^{\square}=y / b=y^{\prime} \\
\alpha^{\square}\left(x^{\square}, y^{\square}\right) \exp (i \omega t)=-U^{-1} \frac{D}{D t} z(x, y, t) \\
\alpha^{\square}\left(x^{\square}, y^{\square}\right)=\exp \left(-i \lambda x^{\square}\right) \alpha\left(x^{\prime}, y^{\prime}\right) \\
\gamma^{\square}\left(x^{\square}, y^{\square}\right) \exp (i \omega t)=\left(\frac{1}{2} \rho U^{2}\right)^{-1}[-2 p(x, y, 0+)]=4 b U^{-1} \frac{D}{D t} \phi(x, y, 0+, t) \\
\gamma^{\square}\left(x^{\square}, y^{\square}\right)=4 \tan \theta \exp \left(-i \lambda x^{\square}\right) \gamma\left(x^{\prime}, y^{\prime}\right) \\
\lambda=2 k \sec ^{2} \theta \\
k=(\omega b / U)
\end{gathered}
$$

The unit of length selected is the half chord $(b)$, in accordance with the generally accepted, two-dimensional notation. The reduced frequency parameters $k$ and $\lambda$ are also defined in accordance with standard notation, namely that used by Biot. ${ }^{16} \lambda$ should not

${ }^{16}$ loc cit. $1,2$. 
be confused with the propagation constant defined by Eq. (3.5) and is henceforth used only in the sense of Eq. (7.7). $\alpha^{\square}$ is the ratio of the (amplitude of the) downwash velocity to the free stream velocity, and $\gamma^{\square}$ is the dimensionless, pressure jump across the wing. In terms of this notation, the substantial time differentiation, cf. Eq. (2.3), is effected by the operator

$$
\frac{D}{D t}(\quad)=(U / 2 b)\left[\frac{\partial}{\partial x^{\square}}(\quad)+2 i k(\quad)\right]
$$

The dimensionless pressure due the downwash distribution

$$
\alpha^{\square}\left(x^{\square}, y^{\square}\right)=\left(y^{\square}\right)^{n} \alpha_{n}^{\square}\left(x^{\square}\right)
$$

is given by Eqs. (7.6b), (2.9b), and (5.4e) as

$$
\begin{aligned}
& \gamma_{n}^{\square}\left(x^{\square}, y^{\square}\right)=4 \tan \theta 1\left(y^{\square}\right) \pi^{-1}\left(\frac{\partial}{\partial x^{\square}}+2 i k\right) \sum_{m=0}^{n}\left(\begin{array}{c}
n \\
m
\end{array}\right) \Gamma(m+1 / 2) \\
& \cdot \int_{0}^{x^{\square}} d \xi \exp (-i \lambda \xi) \alpha_{n}^{\square}\left(x^{\square}-\xi\right) \int_{0}^{y^{\square}} d \eta \eta^{-1 / 2}\left(y^{\square}-\eta\right)^{n-m} k_{m}(2 \xi \tan \theta, \eta)
\end{aligned}
$$

The corresponding lift and moment coefficients, as given by Eqs. (7.1)-(7.3) and (7.6) may be written

$$
\begin{gathered}
C_{L_{n}}=\int_{0}^{1} d x^{0} \gamma_{n}^{\square}(x) \\
C_{M_{n}}=\int_{0}^{1} d x\left(\frac{1}{2}-x\right)^{0} \gamma_{n}^{\square}(x) \\
C_{l_{\Delta}}=\frac{1}{2} \int_{0}^{1} d x^{1} \gamma_{n}^{\square}(x) \\
{ }^{i} \gamma_{n}^{\square}(x)=A^{-1} \int_{0}^{A} d y y^{i} \gamma_{n}^{\square}(x, y)
\end{gathered}
$$

Substituting $\gamma_{n}^{\square}$ from Eq. (7.11) in Eq. (7.15) and integrating by parts yields

$$
\begin{gathered}
{ }^{i} \gamma_{n}^{\square}(x)=4 \tan \theta\left(\frac{\partial}{\partial x}+2 i k\right) \sum_{m=0}^{n}\left(\begin{array}{c}
n \\
m
\end{array}\right) \Gamma(m+1 / 2) \\
\cdot \int_{0}^{x} d \xi \exp (-i \lambda \xi) \alpha_{n}^{\square}(x-\xi) k_{m n}^{i}(2 \xi \tan \theta) \\
k_{m n}^{0}(x)=\pi^{-1}(n-m+1)^{-1} A^{n-m} \int_{0}^{A} d y y^{-1 / 2}\left(1-A^{-1} y\right)^{n-m+1} k_{m}(x, y) \\
k_{m n}^{1}(x)=\pi^{-1}(n-m+2)^{-1}(n-m+1)^{-1} A^{n-m} \int_{0}^{A} d y y^{-1 / 2} \\
\cdot\left(1-A^{-1} y\right)^{n-m+2} k_{m}(x, y)
\end{gathered}
$$


In order to evaluate the $k_{m n}^{i}(x)$, the restriction $A^{\prime} \geq 2$ will be imposed (recalling, cf. section 6 , that the end results for the force coefficients will be valid for $A^{\prime} \geq 1$ ), whence $2 x^{\square} \tan \theta \leq A$. Accordingly, the upper limit $A$ in the $y$ integrations may be replaced by infinity, by virtue of the step function $1(x-y)$ in $k_{m}(x)$, cf. Eq. (5.3). Then, if $k_{m}(x, y)$ is substituted in Eqs. (7.17) and (7.18), the $y$ integrals may be evaluated in terms of Gamma functions and the $\mu$ inversions effected by CF 571 , whence

$$
\begin{aligned}
k_{m n}^{0,1}(x) & =\pi^{-1 / 2} \Gamma(n-m+1) \sum_{s=0}^{n-m+1,2}(-)^{s} \Gamma^{-1}(n-m-s+2,3) \Gamma^{-1}(s+1) \\
\cdot & \Gamma^{-1}\left[\frac{1}{2}(m+s+1)\right] \Gamma\left(s+\frac{1}{2}\right) A^{n-m-s}\left(\frac{x}{2 \kappa}\right)^{(m+s) / 2} J_{(m+s) / 2}(\kappa x) 1(x)
\end{aligned}
$$

Substituting this result in Eq. (7.16) yields

$$
\begin{aligned}
{ }^{i} \gamma_{n}^{\square}(x) & =4 \tan \theta \mathbf{1}(x) \pi^{-1 / 2} \Gamma(n+1)\left(\frac{\partial}{\partial x}+2 i k\right) \sum_{m=0}^{n} \Gamma\left(m+\frac{1}{2}\right) \Gamma^{-1}(m+1) \\
& \cdot \sum_{i=0}^{n-m+1+i}(-)^{s} \Gamma^{-1}(n-m-s+2+i) \Gamma^{-1}(s+1) \Gamma^{-1}\left[\frac{1}{2}(m+s+1)\right] \\
& \cdot \Gamma\left(s+\frac{1}{2}\right) A^{n-m-s} \int_{0}^{x} d \xi \exp (-i \lambda \xi)\left(\xi \sin \frac{\theta}{k}\right)^{(m+s) / 2} \\
\cdot & J_{(m+s) / 2}(\lambda \xi \sin \theta) \alpha_{n}(x-\xi), \quad i=0,1
\end{aligned}
$$

8. Downwash independent of $y$. In principle, any practical downwash distribution may be expanded in terms of the form $y^{n} \alpha_{n}(x)$, the only restriction being that the spanwise dependence be sufficiently continuous to allow a power series expansion to the desired accuracy. In practice, the evaluation of the terms in Eq. (7.20) and the integrations indicated by Eqs. (7.12)-(7.14) will be exceedingly cumbersome for large $n$. The simplest case is that of a downwash which exhibits no spanwise dependence, i.e. $n=0$, which will be treated in this section. In this case only $C_{L}$ and $C_{M}$ are of interest, so that only ${ }^{0} \gamma_{0}^{\square}$ is required.

Setting $n=0$ in Eq. (7.20) and dispensing with the indices yields

$$
\begin{aligned}
\gamma^{\square}(x)= & 4 \tan \theta 1(x)\left(\frac{\partial}{\partial x}+2 i k\right) \int_{0}^{x} d \xi \exp (-i \lambda \xi) \alpha^{\square}(x-\xi) \\
& {\left[J_{0}(\lambda \xi \sin \theta)-\left(\lambda A^{\prime} \sin \theta\right)^{-1} \sin (\lambda \xi \sin \theta)\right] }
\end{aligned}
$$

The first term in the square brackets corresponds to the two dimensional result, ${ }^{17}$ and the second term may be interpreted as the correction for finite aspect ratio. Moreover, the two dimensional results (for lift and moment coefficients) may be expressed in terms of a set of integrals (required for $n=0,1,2,3$ ) of the form ${ }^{18}$

$$
f_{n}=f_{n}(\lambda, \theta)=\int_{0}^{1} \xi^{n} \exp (-i \lambda \xi) J_{0}(\lambda \xi \sin \theta) d \xi
$$

${ }^{17} \mathrm{~J}$. W. Miles, The aerodynamic forces on an oscillating airfoil at supersonic speeds, J. Aero Sci. 14, 351-359 (1947), Eqs. (9) and (12).

${ }^{18}$ loc. cit. 2 , p. 2. 
Accordingly, the two dimensional results are applicable to the rectangular wing ( $A$ cot $\theta \geq 1$ ) if the $f_{n}$ are replaced by

$$
\begin{gathered}
f_{n}\left(\lambda, \theta, A^{\prime}\right)=f_{n}(\lambda, \theta)-\left(\lambda A^{\prime} \sin \theta\right)^{-1} f_{n}^{*}(\lambda, \theta) \\
f_{n}^{*}(\lambda, \theta)=\int_{0}^{1} \xi^{n} \exp (-\imath \lambda \xi) \sin (\lambda \xi \sin \theta) d \xi
\end{gathered}
$$

Integrating Eq. (8.4) twice by parts yields the convenient recursion formula

$$
\begin{gathered}
\lambda^{2} \cos ^{2} \theta f_{n}^{*}=\{[(\lambda \sin \theta) \cos (\lambda \sin \theta)+(i \lambda-n) \sin (\lambda \sin \theta)] \exp (-i \lambda) \\
\left.-\delta_{n}^{0}(\lambda \sin \theta)\right\}-2 i n \lambda f_{n-1}^{*}+n(n-1) f_{n-2}^{*}
\end{gathered}
$$

As a simple example, and as an additional check on the results, the lift coefficient for a flat, rectangular wing at an angle of attack $\alpha_{0}$ in a steady flow (so $k=\lambda=0$ ) will be calculated. Setting $\alpha^{\square}(x) \equiv \alpha_{0}$ and $k=\lambda=0$ in Eq. (8.1) yields

$$
\gamma^{\square}(x)=\left(4 \alpha_{0} \tan \theta\right)\left[1-\left(x / A^{\prime}\right)\right]
$$

The corresponding lift coefficient is given by Eq. (7.12b) as

$$
C_{L}=\left(4 \alpha_{0} \tan \theta\right)\left[1-\left(2 A^{\prime}\right)^{-1}\right]
$$

in agreement with the well known result of Busemann. ${ }^{19}$

9. Moment due to roll. As an example of an antisymmetric problem, the moment due to roll will be calculated. If the angular velocity in roll about the midspan line is $p$, the dimensionless downwash distribution is given by

$$
\alpha^{\square}(x, y)=1(x, y)(p b A / U)\left(A^{-1} y-1\right)
$$

The required pressure function for the determination of the rolling moment, cf. Eq. (7.14b), is therefore $-{ }^{1} \gamma_{0}^{\square}+{ }^{1} \gamma_{1}^{D} / A$; calculating this quantity from Eq. (7.23), substituting in Eq. (7.14b), and integrating the resulting terms in $J_{1}(\lambda \xi \sin \theta)$ and $\cos$ $(\lambda \xi \sin \theta)$ by parts, the integrals may be evaluated in terms of the $f_{n}$ and $f_{n}^{*}$ of Eqs. (8.2) and (8.4) with the result

$$
\begin{aligned}
C_{l_{\nu}}=\partial C_{l} / \partial(p b A / U) \\
=-2 / 3\left[(\tan \theta+2 i k) f_{0}-2 i k f_{1}\right]+2\left(\lambda A^{\prime} \sin \theta\right)^{-1}\left[(\tan \theta+2 i k) f_{0}^{*}\right. \\
\left.-2 i k f_{1}^{*}\right]-2\left(\lambda A^{\prime} \sin \theta\right)^{-2}\left[(\tan \theta+2 i k) f_{0}\right. \\
+2 k \sec \theta(2 k \csc \theta-2 i \cos \theta-i \sec \theta) f_{1}-4 k^{2} \sec \theta \csc \theta f_{2} \\
\left.-\tan \theta \exp (-i \lambda) J_{0}(\lambda \sin \theta)\right]-\left(\lambda A^{\prime} \sin \theta\right)^{-3}\left\{2(\tan \theta+2 i k) f_{0}^{*}\right. \\
+2 k \sec \theta\left[4 k \csc \theta\left(1+\cos ^{2} \theta\right)-3 i \cos \theta-i \sec \theta\right] f_{1}^{*} \\
\left.-4 k^{2} \sec \theta \csc \theta\left(1+\cos ^{2} \theta\right) f_{2}^{*}-\tan \theta \exp (-i \lambda) \sin (\lambda \sin \theta)\right\}
\end{aligned}
$$

${ }^{19} \mathrm{~A}$. Busemann, Infinitesimale kegelige Überschallströmung, Jahrbuch der Luftfahrtforschung 7B, 105-121 (1943). 
10. Arbitrary time dependence. While the present paper has been focused on the case of harmonic time dependence, the results are, in principle, applicable to the calculation of the forces on the wing due to a downwash with an arbitrary time dependence, by virtue of the linearization and the well known Fourier theorem.

An alternative approach to the treatment of arbitrary time dependence would be to use the response to a step function as the basic solution. In this case, the dimensionless downwash is presumed to be of the form

$$
\alpha^{\square}(x, y, t)=\alpha^{\square}(x, y) \mathbf{1}\left[t-t_{0}(x, y)\right]
$$

which is to say that the disturbance at $(x, y)$ arises abruptly at $t_{0}(x, y)$. Suppose that the solution is placed in the form

$$
\gamma^{\square}(x, y, t)=\int_{S} d \xi \int d \eta \mathbf{a}\left[x-\xi, y-\eta, t-t_{0}(\xi, \eta)\right] \alpha^{\square}(\xi, \eta)
$$

where $\gamma^{\square}$ is the dimensionless pressure jump. Suppose further that the harmonic time dependence problem is written

$$
\begin{gathered}
\alpha^{\square}(x, y, t)=\alpha^{\square}(x, y) \exp (i \omega t) \\
\gamma^{\square}(x, y, t)=\exp (i \omega t) \int_{S} d \xi \int d \eta \mathbf{k}(x-\xi, y-\eta, \omega) \alpha^{\square}(\xi, \eta)
\end{gathered}
$$

Then, by virtue of the Fourier representation of the step function of Eq. (10.1), $\mathbf{a}$ and $\mathbf{k}$ are related by

$$
\mathbf{a}(x, y, t)=(2 \pi)^{-1} \lim _{\epsilon \rightarrow 0+} \int_{-\infty}^{\infty} d \omega(\epsilon+i \omega)^{-1} \mathbf{k}(x, y, \omega) \exp (i \omega t)
$$

which is to say that the indicial admittance a is the Bromwich integral of the Green's function $\mathbf{k}$. Accordingly, the two approaches are complementary, and, having solved for $\mathbf{k}$, as in the present paper, a follows from Eq. (10.5). The application to the gust loading of a rectangular wing has been given in a separate paper. ${ }^{20}$

11. Numerical results. Numerical results for the lifts and moments on a rectangular airfoil due to plunging and pitching oscillations have been obtained and are available elsewhere. ${ }^{21}$

12. Related papers (added in proof). The special case of section 7 has been treated independently by Stewart and $\mathrm{Li}^{22}$ and by Stewartson. ${ }^{23}$ The results presented in the latter paper are in agreement with those presented herein, whereas those of the former are not. Moreover, the result (8.1) has been checked by the author, using still a fourth method. ${ }^{24}$ Hence, it appears that the general method developed by Stewart and $\mathrm{Li}$ may be in error.

${ }^{20} \mathrm{~J}$. W. Miles, Transient loading of supersonic rectangular airfoils, J. Aero. Sci., 17, 647-652 (1950).

${ }^{21} \mathrm{~J}$. W. Miles and Irven Naiman, Aerodynamic derivatives for oscillating rectangular airfoils at supersonic speeds, U.S.N.O.T.S. Tech. Memo RRB-32, Inyokern, Calif. (1949).

${ }^{22} \mathrm{H}$. J. Stewart and T. Y Li, Periodic motions of a rectangular wing at supersonic speed, J. Aero. Sci., 17, 529-538 (1950).

${ }^{23} \mathrm{~K}$. Stewartson, On the linearized potential theory of unsteady supersonic motion, Q. J. Mech. and Appl. Math. 3, 182-199 (1950).

${ }^{24} \mathrm{~J}$. W. Miles, On the reduction of unsteady supersonic flow problems to steady flow problems, J. Aero. Sci. 17, 64 (1950). 